\title{
Aiming for a Direct Hit: Combining Molecular Imaging with Targeted Cancer Therapy
}

$O$

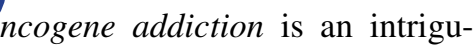
ing new term that has emerged in the lexicon of cancer biology (1). This term implies that tumor cells may have an extraordinary, almost complete dependence on the function of one or more genes (oncogenes) for their survival, whereas these same genes expressed in normal cells are not absolutely essential. The human epidermal growth factor receptor type 2 (HER2) is one such oncogene that encodes a transmembrane receptor tyrosine kinase overexpressed in about

See Page 1131

$20 \%$ of breast cancers, primarily because of gene amplification (2). HER2 overexpression confers an aggressive tumor phenotype that correlates with hormone insensitivity (3), chemotherapy resistance (4), and poor long-term survival (5). Trastuzumab (Herceptin; Roche Pharmaceuticals) is a humanized $\mathrm{IgG}_{1}$ monoclonal antibody approved for the treatment of HER2-amplified metastatic breast cancer and as an adjuvant treatment for early-stage disease (6). The mechanisms through which trastuzumab exerts its antitumor effects are not completely understood, but an important predictor of response is tumor HER2 overexpression (7). HER2 positivity is assessed in a biopsy sample of primary breast cancer by immu-

Received Jan. 3, 2009; revision accepted Jan. 7, 2009

For correspondence or reprints contact: Raymond M. Reilly, University of Toronto, 144 College St. Toronto, Ontario, Canada.

E-mail: raymond.reilly@utoronto.ca

COPYRIGHT @ 2009 by the Society of Nuclear

Medicine, Inc.

DOI: 10.2967/jnumed.108.060772 nohistochemical staining or probing for HER2 gene amplification by fluorescence in situ hybridization. There is generally good concordance in HER2 positivity between primary and metastatic lesions, but some discrepancies have been noted (8). Patients with HER2-amplified tumors (immunohistochemical staining $3+$ or HER2-tochromosome-17 control gene sequence ratios $>2.2$ ) are expected to benefit most from trastuzumab (9).

A novel alternative strategy to interfere with the function of the HER2 oncogene is the use of heat-shock protein (Hsp) inhibitors, in particular those aimed at Hsp90: 17(allylamino)17-demethoxygeldanamycin (17-AAG; tanespimycin) and its orally bioavailable analog, 17(dimethlyaminoethylamino)17-demethoxygeldanamycin (17-DMAG). Hsp90 is a molecular chaperone that complexes with Hsp70 and Hsp40, as well as with cochaperone proteins (p23 and $\mathrm{p} 50^{\mathrm{Cdc} 37}$ ), to mediate the correct folding of numerous proteins in the cell, thereby protecting them from routing to the proteasome for degradation (10). 17-AAG and 17-DMAG block the function of Hsp90 by preventing its conversion to the active adenosine triphosphate-bound form (11). Hsp90 inhibitor treatment of breast cancer is based on the premise that the cell-surface expression of HER2 can be diminished by rerouting newly synthesized receptors to the proteasome for proteolytic destruction. Reliance on Hsp90 may in itself represent a form of oncogene addiction (10). Moreover, there is evidence that Hsp90 permits recycling of internalized HER2 back to the cell surface, thus preserving overexpression (12). Because trastuzumab has promoted $H E R 2$ internalization in some studies (7), its combined use with Hsp90 inhibitors would be complemen- tary and synergistic; in fact, one clinical trial has examined this combination for treatment of HER2-positive breast cancer, with promising results (13).

Fundamental to the success of any therapeutic strategy aimed at disrupting the influence of the HER2 oncogene are an accurate assessment of the level of HER2 density in tumors and the ability to sensitively measure a treatment effect on HER2 expression. In this issue of The Journal of Nuclear Medicine, Kramer-Marek et al. report that small-animal PET with ${ }^{18}$ F-labeled $\mathrm{Z}_{\text {HER2:342 Affibody (Affibody AB) spe- }}$ cific for $H E R 2$ can sensitively provide information on these 2 phenomena (14). Affibody is a new class of targeting vehicle generated from a phage display library of Staphylococcus protein A variants in which substitutions were made to a 13-amino-acid sequence within the Z-domain (15). Initial panning of the library for binding to the HER2 extracellular domain yielded $\mathrm{Z}_{\text {HER2:4 }}$ Affibody with good specificity but relatively low affinity (dissociation constant, $50 \mathrm{nM}$ ) (16). Subsequent modifications and rescreening (affinity maturation) identified the extremely high affinity $\mathrm{Z}_{\text {HER2:324 }}$ Affibody (dissociation constant, 22 pM) used in this study (17). Successful imaging of HER2-overexpressing human tumor xenografts in mice has been achieved with Affibody labeled with

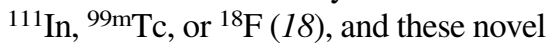
radiopharmaceuticals were recently studied for the first time in humans (19). Kramer-Marek et al. showed that the exquisite affinity of the ${ }^{18} \mathrm{~F}-Z_{\text {HER2:324 }}$ Affibody for HER2 provided excellent tumor uptake $(\leq 21 \%$ injected dose $[\% \mathrm{ID}] / \mathrm{g}$ ) whereas the low molecular weight $(6 \mathrm{kDa})$ caused rapid elimination from the blood. The resulting tumor-toblood ratios exceeded 20:1 at only $1-2 \mathrm{~h}$ after injection, thus making it feasible 
to use the 110-min half-life positronemitter, ${ }^{18} \mathrm{~F}$, as a radiolabel.

There was a direct relationship between uptake of ${ }^{18} \mathrm{~F}-\mathrm{Z}_{\text {HER2:324 }}$ Affibody and tumor HER2 density in a panel of 5 subcutaneous human breast cancer xenografts in athymic mice, except for MCF-7/clone18 tumors. Uptake was lower for MCF-7/ clone18 tumors than for BT-474 xenografts $(12 \% \mathrm{ID} / \mathrm{g}$ vs. $20 \% \mathrm{ID} / \mathrm{g}$, respectively), despite 1.5-fold higher HER2 expression measured ex vivo by enzyme-linked immunosorbent assay. MCF-7/clone18 is a HER2 genetransfected variant of $\mathrm{MCF}-7$ cells, which naturally have low HER2 density. This discrepancy was attributed to possible differences in the microenvironment of MCF-7/clone18 xenografts that may have affected the delivery of ${ }^{18} \mathrm{~F}-\mathrm{Z}_{\text {HER 2:324 }}$ Affibody. Interestingly, we recently reported that strong associations between tumor HER2 density and uptake of ${ }^{111}$ In-labeled trastuzumab in a panel of $5 H E R 2$-positive breast cancer xenografts in athymic mice were obtained only if tumor uptake was corrected for nonspecific IgG localization or circulating blood-pool radioactivity, factors that presumably reflect differences in the tumor microenvironment (20). Reasonably good correlations between epidermal growth factor receptor density and the accumulation of ${ }^{64} \mathrm{Cu}$-labeled anti-epidermal growth factor receptor cetuximab antibodies in epidermal growth factor receptor-positive xenografts in mice have similarly been described (21). However, Aerts et al. (22) have reported disparities in uptake of ${ }^{89} \mathrm{Zr}$-labeled cetuximab by tumors and epidermal growth factor receptor density, again possibly due to differences in tumor physiology that control radiopharmaceutical delivery. Kramer-Marek et al. (14) visualized all breast cancer xenografts by small-animal PET using ${ }^{18} \mathrm{~F}-\mathrm{Z}_{\text {HER2:324 }}$ Affibody, but the tumor signal ranged from low for MCF-7 xenografts to intermediate for MCF-7/clone18 tumors and strong for BT-474 tumors.

The avid accumulation of ${ }^{18} \mathrm{~F}$ $\mathrm{Z}_{\text {HER 2:324 Affibody in the MCF-7/ }}$ clone18 and BT-474 xenografts provided an opportunity to interrogate the effects of 17-DMAG Hsp90 inhibitor therapy on tumor HER2 expression. Tumor-bearing mice received a baseline PET scan and were then treated intravenously with 4 doses of $40 \mathrm{mg} /$ $\mathrm{kg}$ of 17-DMAG over a 60 -h period, followed by repeated imaging $12 \mathrm{~h}$ after the final dose. Region-of-interest analysis revealed a $71 \%$ and $33 \%$ significantly lower uptake of ${ }^{18} \mathrm{~F}$ -

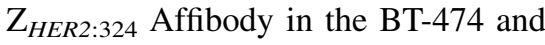
MCF-7/clone18 tumors, respectively, after 17-DMAG treatment. There was virtually complete agreement between decreased tumor uptake of ${ }^{18} \mathrm{~F}$ $\mathrm{Z}_{\text {HER2:324 }}$ Affibody and ex vivo analysis of tumor HER2 expression by enzyme-linked immunosorbent assay and Western blot. Unexpectedly, immunohistochemical staining for HER2 (HercepTest score; Dako) in the explanted tumors did not reveal decreases in HER2 positivity after 17-DMAG treatment, indicating that this technique does not appear to have the sensitivity required to detect these changes. We have also recently found that immunohistochemical staining does not have sufficient sensitivity to detect HER2 downregulation in athymic mice bearing subcutaneous human breast cancer xenografts treated with trastuzumab, whereas micro-SPECT with ${ }^{111}$ In-labeled pertuzumab (a HER2 antibody that recognizes an epitope different from trastuzumab) was capable of monitoring such changes (23). Imaging of decreased tumor HER2 expression caused by Hsp90 inhibition was first reported by Smith-Jones et al. using ${ }^{68}$ Ga-labeled trastuzumab $\mathrm{F}\left(\mathrm{ab}^{\prime}\right)_{2}$ fragments (24); this approach proved to be an earlier predictor of tumor response to 17 -AAG therapy than was ${ }^{18} \mathrm{~F}-\mathrm{FDG}$ PET (25).

It is important to appreciate that the effectiveness of 17-AAG in downregulating tumor $H E R 2$ expression in clinical trials is most commonly measured by Western blot analysis of readily accessible peripheral blood lymphocytes for Hsp90, Hsp70, or client proteins (e.g., RAF-1, cdk4, or LCK) rather than by sampling tumors directly (26). These biomarkers, although convenient, have yielded inconsistent results that do not appear to correlate with tumor response $(13,26,27)$. Molecular imaging using ${ }^{18} \mathrm{~F}-\mathrm{Z}_{\text {HER2:324 }}$ Affibody may provide a more sensitive, direct, and highly feasible means of evaluating tumor response in situ to these new therapies; it could also be invaluable in optimizing the dose. Phase I clinical trials of 17-AAG have used conventional doseescalation protocols to reach the maximally tolerated dose for subsequent phase II studies, yet this may not be the lowest or optimal dose required to block Hsp90. These drugs are associated with significant toxicities, including hepatotoxicity $(26,27)$, and thus, identifying the minimum dose that is effective in blocking Hsp90 would be desirable. PET with ${ }^{18} \mathrm{~F}-\mathrm{Z}_{\text {HER2:324 }}$ Affibody could probe the downstream effect of increasing doses of 17-AAG or 17-DMAG in patients enrolled in trials of Hsp90 inhibitors for the treatment of HER2-positive tumors. Evaluation of the kinetics of HER2 downregulation by Hsp90 inhibitors through repeated imaging either preclinically or clinically would be facilitated by the rapid biologic elimination of ${ }^{18} \mathrm{~F}-\mathrm{Z}_{\text {HER2:324 }}$ Affibody and the short physical half-life of the radionuclide. The results of such molecular imaging of HER2 expression may be particularly profound for combinations of trastuzumab and 17AAG (13), in which there may be dramatic downregulation due to the potential synergy between these 2 drugs as discussed earlier. Molecular imaging is a sensitive tool that clearly has an important future role to play in predicting and monitoring response to new targeted cancer therapies that aim to exploit oncogene addiction (28). The study by Kramer-Marek et al. (14) stimulates our imagination about what could be achieved by combining these 2 exciting and continually evolving fields.

Raymond M. Reilly University of Toronto Toronto, Ontario, Canada 


\section{REFERENCES}

1. Weinstein IB, Joe A. Oncogene addiction. Cancer Res. 2008;68:3077-3080.

2. Owens MA, Horten BC, Da Silva MM. HER2 amplification ratios by fluorescence in situ hybridization and correlation with immunohistochemistry in a cohort of 6556 breast cancer tissues. Clin Breast Cancer. 2004;5:63-69.

3. Konecny G, Pauletti G, Pegram M, et al. Quantitative association between HER2/neu and steroid hormone receptors in hormone receptorpositive primary breast cancer. J Natl Cancer Inst. 2003;95:142-153.

4. Colomer R, Montero S, Lluch A, et al. Circulating HER2 extracellular domain and resistance to chemotherapy in advanced breast cancer. Clin Cancer Res. 2000;6:2356-2362.

5. Slamon DJ, Clark GM, Wong SG, Levin WJ, Ullrich A, McGuire WL. Human breast cancer: correlation of relapse and survival with amplification of the HER-2/neu oncogene. Science. 1987; 235:177-182.

6. Smith I, Procter M, Gelber RD, et al. 2-year follow-up of trastuzumab after adjuvant chemotherapy in HER2-positive breast cancer: a randomized controlled trial. Lancet. 2007;369:29-36.

7. Baselga J, Albanell J. Mechanism of action of antiHER2 monoclonal antibodies. Ann Oncol. 2001; 12(suppl 1):S35-S41.

8. Zidan J, Dashkovsky I, Stayerman C, Basher W, Cozacov C, Hadary A. Comparison of HER-2 overexpression in primary breast cancer and metastatic sites and its effect on biological targeting of metastatic disease. Br J Cancer. 2005;93:552-556.

9. Wolff AC, Hammond EH, Schwartz JN, et al. American Society of Clinical Oncology/College of American Pathologists guideline recommendations for human epidermal growth factor receptor 2 testing in breast cancer. J Clin Oncol. 2007;25:118-145.
10. Workman P, de Billy E. Putting the heat on cancer. Nat Med. 2007;13:1415-1417.

11. Isaacs JS, Xu W, Neckers L. Heat shock protein 90 as a molecular target for cancer therapeutics. Cancer Cell. 2003;3:213-217.

12. Austin CD, De Mazière AM, Pisacane PI, et al. Endocytosis and sorting of ErbB2 and the site of action of cancer therapeutics trastuzumab and geldanamycin. Mol Biol Cell. 2004;15:5268-5282.

13. Modi S, Stopeck AT, Gordon MS, et al. Combination of trastuzumab and tanespimycin (17-AAG, KOS-953) is safe and active in trastuzumabrefractory HER-2-overexpressing breast cancer: a phase I dose-escalation study. $J$ Clin Oncol. 2007;25:5410-5417.

14. Kramer-Marek G, Kiesewetter DO, Capala J. Changes in HER2 expression in breast cancer xenografts after therapeutic intervention can be quantified using PET and ${ }^{18} \mathrm{~F}$-labeled Affibody molecules. J Nucl Med. 2009;50:1131-1139.

15. Nord K, Gunneriusson E, Ringdahl J, Ståhl S, Uhlén M, Nygren P-Å. Binding proteins selected from combinatorial libraries of an $\alpha$-helical bacterial receptor domain. Nat Biotechnol. 1997;15:772-777.

16. Wikman M, Steffen A-C, Gunneriusson E, et al. Selection and characterization of HER2/neu-binding Affibody ligands. Protein Eng Des Sel. 2004;17: 455-462.

17. Orlova A, Magnusson M, Eriksson T, et al. Effect of affinity maturation on biodistribution of radioiodinated anti-HER2 Affibody molecules [abstract]. Eur J Nucl Med Mol Imaging. 2005;32(suppl 1):S78.

18. Tolmachev V. Imaging of HER-2 overexpression in tumors for guiding therapy. Curr Pharm Des. 2008;14:2999-3019.

19. Baum RP, Orlova A, Tolmachev V, Feldwisch J. A novel molecular imaging agent for diagnosis of recurrent HER2 positive breast cancer: first time in human study using an indium-111- or gallium-68 labeled Affibody molecule [abstract]. Eur J Nucl Med Mol Imaging. 2006;33(suppl 2):S91.

20. McLarty K, Cornelissen B, Scollard DA, Done SJ, Chun K, Reilly RM. Associations between the uptake of ${ }^{111}$ In-DTPA-trastuzumab, HER2 density and response to trastuzumab (Herceptin) in athymic mice bearing subcutaneous human tumour xenografts. Eur J Nucl Med Mol Imaging. 2009;36:81-93.

21. Cai W, Chen K, He L, Cao Q, Koong A, Chen X. Quantitative PET of EGFR expression in xenograft-bearing mice using ${ }^{64} \mathrm{Cu}$-labeled cetuximab, a chimeric anti-EGFR monoclonal antibody. Eur $J$ Nucl Med Mol Imaging. 2007;34:850-858.

22. Aerts HJWL, Dubois L, Perk L, et al. Disparity between in vivo EGFR expression and ${ }^{89} \mathrm{Zr}$-labeled cetuximab uptake assessed with PET. J Nucl Med. 2009;50:123-131.

23. McLarty K, Cornelissen B, Scollard D, Reilly RM. ${ }^{111} \mathrm{In}$-pertuzumab is a sensitive probe of trastuzumabmediated HER2 downregulation in vitro and in vivo [abstract]. J Nucl Med. 2008;49(suppl):105P.

24. Smith-Jones PM, Solit DB, Akhurst T, Afroze F, Rosen N, Larson SM. Imaging the pharmacodynamics of HER-2 degradation in response to Hsp90 inhibitors. Nat Biotechnol. 2004;22:701-706.

25. Smith-Jones PM, Solit D, Afroze F, Rosen N, Larson SM. Early tumor response to Hsp90 therapy using HER2 PET: comparison with ${ }^{18}$ F-FDG PET. J Nucl Med. 2006;47:793-796.

26. Banerji U, O'Donnell A, Scurr M, et al. Phase I pharmacokinetic and pharmacodynamic study of 17-allylamino, 17-demethoxygeldanamycin in patients with advanced malignancies. J Clin Oncol. 2005;18:4152-4161.

27. Goetz MP, Toft D, Reid J, et al. Phase I trial of 17allylamino-17-demethoxygeldanamycin in patients with advanced cancer. J Clin Oncol. 2005;23:1078-1087.

28. McLarty K, Reilly RM. Molecular imaging as a tool for personalized and targeted anticancer therapy. Clin Pharmacol Ther. 2007;81:420-424. 